\title{
Influence of M. tibialis anterior fatigue on the walk-to-run and run-to-walk transition in non-steady state locomotion
}

\author{
Segers $\mathrm{V}^{\mathrm{a}}$, Lenoir $\mathrm{M}^{\mathrm{a}}$, Aerts $\mathrm{P}^{\mathrm{a}, \mathrm{b}}$, De Clercq $\mathrm{D}^{\mathrm{a}, *}$ \\ ${ }^{a}$ Department of Movement and Sport Sciences, Faculty of Medicine and Health Sciences, Ghent University, \\ Watersportlaan 2, B-9000 Gent, Belgium \\ ${ }^{\mathrm{b}}$ Department of Biology Laboratory for Functional Morphology, University of Antwerp, Belgium
}

Received 23 November 2005; received in revised form 22 June 2006; accepted 8 July 2006

\begin{abstract}
The purpose of this study was to examine the influence of muscular fatigue of tibialis anterior (TA) on the walk-to-run transition (WRT) and run-to-walk transition (RWT) when speed is altered at different constant accelerations $\left(a=0.01,0.07\right.$ and $\left.0.05 \mathrm{~m} \mathrm{~s}^{-2}\right)$. Twenty women (height: $168.9 \pm 3.36 \mathrm{~cm}$ ) performed WRTs and RWTs on a motor-driven treadmill, before and after a protocol inducing muscular fatigue of the TA.

WRT-speed decreased after TA fatigue whereas RWT-speed did not change except during the intermediate deceleration. Integrated EMG (iEMG) of the activity burst of TA around heel contact was examined in the last steps before transition, the transition step and the first steps after transition. iEMG increased before WRT, then decreased after transition to running. In the RWT the opposite was observed: iEMG increased after RWT, then decreased with decreasing walking speed. After inducing fatigue in the TA, there was a decrease in iEMG in the WRT whereas no influence of fatigue was found on iEMG in the RWT.

As a result of TA fatigue, WRT occurred at a lower speed, probably to avoid over-exertion of the TA. This indicates that the TA is a likely determinant of WRT as previously reported. The RWT, on the other hand, was not altered following TA fatigue, which would indicate that WRT and RWT are determined by different factors.
\end{abstract}

(C) 2006 Elsevier B.V. All rights reserved.

Keywords: Gait transition; M. tibialis anterior; EMG; Biomechanics

\section{Introduction}

Increasing speed results in a change from walking to running. Decreasing speed on the other hand results in the opposite transition from running to walking [1-4]. Both transitions occur at a preferred speed [5]. Nevertheless, it is not yet entirely clear why humans prefer that specific speed to change from one mode to another [6].

One of the most obvious reasons for the transition is metabolic cost, that is, a change to another type of locomotion reduces oxygen consumption. Regarding the relationship between metabolic cost and transition, conflicting results have been found. Some studies suggested that the walk-to-run transition (WRT) is closely linked to the

\footnotetext{
* Corresponding author. Tel.: +32 926463 22; fax: +329 2646484 .

E-mail address: Dirk.DeClercq@UGent.be (D.C. D).
}

minimization of metabolic cost $[7,8]$. Others found evidence to reject this energy optimisation hypothesis [9-13]. This contradiction can be partly explained by the difficulties in directly measuring the metabolic cost $[6,7]$.

In the absence of a clear metabolic trigger, it is unclear why subjects perceive walking as "harder" than running at transition speed $[10,14]$. Subjects might use information from peripheral receptors, from the activity in the neural networks controlling locomotion, as well as from previous experience, to trigger transition and achieve a more comfortable mode of locomotion [15]. This is supported by evidence showing that perceived effort during lowintensity exercise (estimated by the rate of perceived exertion) originates from motor outflow commands to muscles (quantified by muscle activation) and, to a lesser degree, from the afferent information of the actual force developed by the muscles [16]. 
The larger, proximal muscle groups are not activated near their maximal level when walking or running at a speed close to WRT-speed. Therefore, muscular activation level and muscular stress level is low [2,3]. The smaller and distal tibialis anterior muscle (TA), however, is activated near its maximum capacity and experiences high muscular stress around WRT-speed [2]. The amplitude of peak EMG of the TA increased with increasing walking speed but suddenly decreased after transition [2]. Also, at WRT-speed, critical levels of ankle angular velocities and accelerations are reached $[3,5]$. This apparently crucial role of the TA led to the hypothesis that the WRT is determined at the ankle region $[2,5]$. EMG of the TA during walking and running has a typical phasic activity pattern with a burst during the eccentric foot plantarflexion movement following heel contact. This eccentric activity may be causing an increased perceived exertion, which would serve as protective mechanism to prevent further damage [17].

Although WRT may be triggered by information arising around the ankle region, this may not necessarily be the case for run-to-walk transition (RWT). The ankle velocity and acceleration change from a lower value in running to a higher value in walking [5]. Therefore, Prilutsky and Gregor [3] suggested that RWT might be controlled by other muscle groups, namely the muscles active during stance (soleus, gastrocnemius and vastus lateralis). The perception of increased effort in these support-related muscles is likely to be required for the acceleration and deceleration of the body's centre of mass and the larger peaks of vertical ground reaction forces [3,18].

The purpose of this study was to examine transition speed and activity of the TA in a protocol with gradually changing speed to investigate the actual transition $\operatorname{step}(\mathrm{s})$ when accelerating across transition speed. By inducing fatigue in the TA, local perceived exertion is expected to increase [19] which then decreases WRT-speed ${ }^{2}$.

The main hypotheses of this study are: (1) WRT occurs at a lower speed following TA fatigue, while RWT remains unaffected, (2) integrated EMG (iEMG) will increase as walking speed increases in the WRT, then decrease after WRT, both before and after TA fatigue and (3) TA activity in the RWT will not be affected by transition nor by TA fatigue.

\section{Materials and methods}

\subsection{Subjects}

Twenty physically active female subjects took part in the present research, having signed informed consent (Table 1). Subjects of height between 1.65 and $1.75 \mathrm{~m}$ were chosen to minimize possible influence of anthropometric values on transition speed [20]. At the time of the study all subjects were free from any disease or injury that could affect the results. The ethical committee of Ghent University Hospital approved the experimental protocol.
Table 1

Subjects characteristics: mean $(X)$ and standard deviation (S.D.) for height, body mass, leg length and age

\begin{tabular}{lcl}
\hline & $X$ & S.D. \\
\hline Height $(\mathrm{cm})$ & 168.9 & 3.4 \\
Weight $(\mathrm{kg})$ & 63.2 & 5.9 \\
Leg length $(\mathrm{cm})$ & 91.4 & 1.8 \\
Age (years) & 24.5 & 2.8 \\
Physical activity $^{\mathrm{a}}$ (h/week) & 2.82 & 0.95 \\
\hline
\end{tabular}

${ }^{\text {a }}$ Sports on competitive level.

\subsection{Treadmill protocol}

The experiment was divided into two sessions. For each session, every subject performed 30 trials divided into six blocks of five trials with a resting period of $30 \mathrm{~s}$ between each block. Each block was characterized by a constant acceleration. This type of protocol with gradually changing speed was chosen because transition is thought to be a process $[4,21]$ with acceleration as an important task constraint [22]. The accelerations were $0.10,0.07,0.05,-0.10,-0.07$ and $-0.05 \mathrm{~m} \mathrm{~s}^{-2}$. Positive and negative accelerations, respectively, caused WRT and RWT. By choosing these values, the acceleration at which WRT-speed probably equals RWTspeed (no hysteresis at $0.07 \mathrm{~m} \mathrm{~s}^{-2}$ ) [22] was included together with distinctly lower $\left(0.05 \mathrm{~m} \mathrm{~s}^{-2}\right)$ and higher $\left(0.10 \mathrm{~m} \mathrm{~s}^{-2}\right)$ values. The blocks were randomly selected for subjects, while alternating positive and negative accelerations. The first block was not incorporated in the calculations but was considered a familiarization trial.

During the first session, all subjects were familiarized with the treadmill by performing treadmill locomotion at different speeds [23] for at least $15 \mathrm{~min}$. The second session began with the fatigue-inducing protocol followed by the treadmill protocol. The time elapsed between the fatigueinducing and treadmill protocol was maximally 2 min to exclude potential recovery of TA.

The actual speed of the treadmill was on-line electronically registered $(5 \mathrm{~Hz})$ and synchronized with video recordings by means of LED's.

\subsection{Fatigue protocol}

Subjects were seated with thighs and trunk strapped to the chair in order to eliminate the undesired use of these segments in the fatigue protocol. A sub-maximal load ( $\pm 70 \%$ of one repeated maximum) was placed on the "Tib Exerciser", a fitness device used to train the TA. Subjects were asked to move the load up and down at a constant speed. This was supervised by an experienced researcher to create a standardized protocol. Subjects performed series of 15 repetitions with a 30 -s break between successive series, until exhaustion was reached. If a series was not completed, subjects were offered a second try after a 1 min break. An adapted Borg scale (scale 1-10) was used to scale localized muscle fatigue [24]. 


\subsection{Instrumentation}

EMG of TA was recorded over $8 \mathrm{~s}$ at a sampling frequency of $1000 \mathrm{~Hz}$ using bipolar electrodes (Noraxon). Data of the TA were rectified, band-pass filtered (5-2000 Hz) and integrated. Hreljac et al. [2] calculated mean and peak 100-ms moving average activation levels and found an abrupt transition related change to a lower value in the peak EMG-values. Activity of TA during the burst around the time of heel contact was examined by integrated EMG (iEMG) in the present study. iEMG was normalized to the value during step 0 (see below) in the WRT and was calculated as the product of magnitude and duration of the burst. In the approach towards transition more intensive activation of TA results in a higher recruitment level of the muscle, which is reflected in iEMG.

Sagittal plane kinematics, focusing on the leg movement, were measured during all trials using a high-speed video camera (JVC DVL9800) at $200 \mathrm{~Hz}$. The moment of initial (heel) and final (toe-off) contact of the foot was determined from the recordings. Step frequency (SF) was calculated as: $1 / \Delta t(\Delta t=$ the time between two successive foot contacts). Step length (SL) was calculated by dividing instant speed of the treadmill by SF. Duty factor (DF) was the ratio of contact-time and total stride time [25], a parameter used to define walking $(\mathrm{DF}>0.5)$ and running $(\mathrm{DF}<0.5)$. The transition step was called step zero ( 0 ) and was defined as the first step with a flight phase when speed was increased (WRT) or the first step with a double stance phase when speed was decreased (RWT). Every step before transition had a negative sign; every step after transition had a positive sign.

From the video images, foot angle (angle between shoe sole and horizontal treadmill surface) was calculated. A marker was placed on the lateral malleolus and on the fifth metatarsal, and standardized tight fitting running shoes were used.

\subsection{Statistics}

All data were stored and analyzed using the SPSS 11.0 package (SPSS inc., Chicago, IL). Descriptive statistics (mean \pm S.D.) were calculated for subjects' characteristics, speed $(v)$, duty factor (DF), step length (SL) and step frequency (SF), rate of perceived exertion (RPE), number of series, iEMG, duration and magnitude of the bursts, amplitude of the EMG-signal and foot angle.

A paired sample $t$-test was used to evaluate the differences in RPE before and after fatigue. The analyses to compare $v$, DF, SL, SF, iEMG, duration and magnitude of the burst were performed in a step-wise protocol. iEMG before fatigue was examined by a repeated measures analysis of variance (ANOVA) with a Bonferroni test to compare the 11 steps (step -5 to step +5 ) pair wise. Two (negative versus positive acceleration) by two (before and
Table 2

Rate of perceived exertion (RPE) on the adapted Borg scale before and after the fatigue protocol and number of series completed during the fatigue protocol

\begin{tabular}{lll}
\hline & $X$ & S.D. \\
\hline RPE & 2.16 & \\
$\quad$ Before fatigue protocol & $9.33^{* *}$ & 0.78 \\
$\quad$ After fatigue protocol & & 0.34 \\
Series & 10.79 & \\
$\quad$ Number of series completed & & 2.58 \\
\hline
\end{tabular}

Mean $X$ and standard deviation S.D. Significant difference between RPE before and after fatigue ${ }^{* *} p<0.01$.

after fatigue) by three (accelerations 0.10, 0.07 and $0.05 \mathrm{~m} \mathrm{~s}^{-2}$ ) repeated measures ANOVAs were performed to test for differences in transition speed. Since an interaction was found between WRT and RWT (Table 2), throughout the rest of the paper, 2 (fatigue) $\times 3$ (accelera(acceleration) repeated measures ANOVAs were performed to test for effects in WRT and RWT for each step (step -5 to step +5 ) separately. The $2 \times 3$ repeated measures ANOVA revealed significant effects of fatigue in step -1 , step 0 and step +1 and not in the preceding or following steps. Therefore, effects of fatigue were reported for these steps only. Effect size was estimated by a squared partial eta $\left(\eta^{2}\right)$ which expresses the amount of variance, as a fraction of the total amount of variance that can be explained by a certain effect (i.e. step or fatigue).

Non-parametric statistics were used to explore differences in foot angle for five randomly selected subjects. A Friedman test was used for differences between walking, running and transition steps (related samples) whereas a Mann-Whitney $U$-test was used for examination of the influence of TA fatigue.

\section{Results}

\subsection{Fatigue protocol}

The perceived exertion of the TA (from an adapted Borg scale) increased significantly during the fatigue protocol (Table 2). After fatigue, subjects were no longer capable of lifting the weight in a controlled manner, even after encouragement and a resting period of $60 \mathrm{~s}$.

\subsection{Transition speed}

After introducing localized muscular fatigue in the TA, WRT-speed was significantly lower in all accelerations $\left(F_{1,14}=35.341, p<0.01, \eta^{2}=0.716\right)$. Rate of acceleration did not affect WRT-speed $\left(F_{2,28}=2.142, p=0.136\right.$, $\left.\eta^{2}=0.133\right)$ or interact with fatigue $\left(F_{1,14}=0.772\right.$, $p=0.472, \eta^{2}=0.052$ ). 
In the RWT-condition, however, there was an interaction between fatigue and acceleration $\left(F_{2,28}=6.725, p<0.001\right.$, $\left.\eta^{2}=0.324\right)$. RWT-speed was increased after fatigue in the intermediate deceleration.

\subsection{EMG before fatigue}

EMG before fatigue was studied in the last steps before and the first steps after transition.

\subsubsection{WRT}

The repeated measures ANOVA revealed significant effects for normalized iEMG between step -1 and step +1 $(p<0.05)$, whereas only few significant differences were found in all other steps. As can be seen in Fig. 1, iEMG increased during the last walking steps before transition, and dropped to a lower value after transition. In the two lowest accelerations, iEMG increased again in the following running steps.
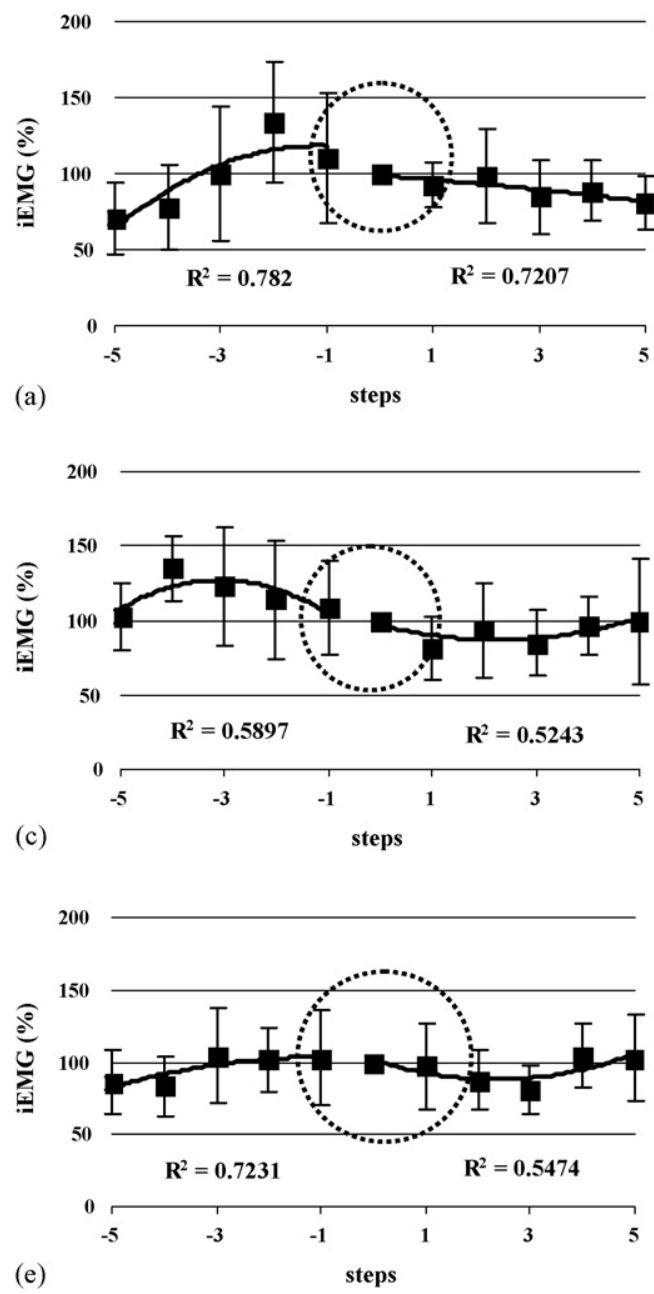

\subsection{2. $R W T$}

Normalized iEMG remained approximately constant in the last running steps, increased at transition to decrease again after some walking steps. iEMG was higher in the first steps after transition in comparison to the last running steps (Fig. 1). After step 3, iEMG decreased to the level observed during the running steps in the two highest decelerations.

\subsection{EMG fatigued}

Changes in EMG activity of the TA around the time of heel contact was examined after fatigue to identify possible underlying mechanisms of the TA as a determinant of WRT and/or RWT.

\subsection{1. $W R T$}

iEMG significantly decreased after muscular fatigue in step -1 and step 0 (Table 4; Fig. 2), with an effect of acceleration in step -1 . In step +1 , on the other hand, fatigue
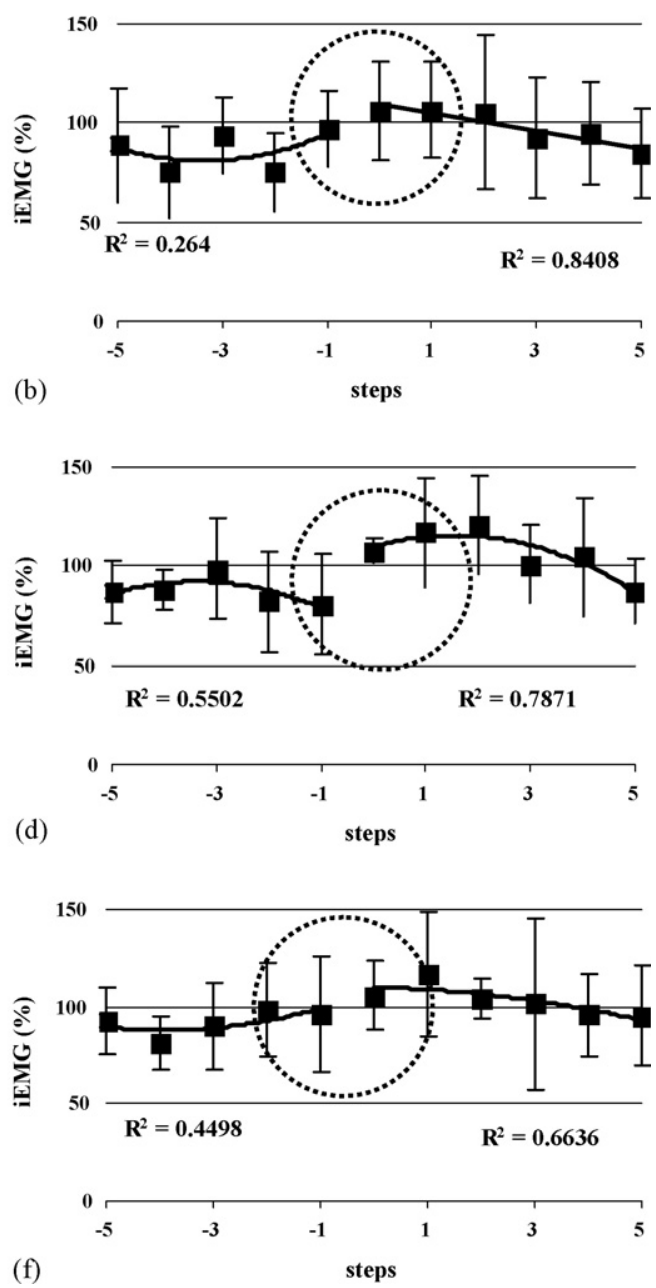

Fig. 1. Evolution of normalized iEMG of the burst of TA activity in proximity of heel contact in the WRT (left side) and RWT (right side), $a=0.10,-0.10,0.07$, $-0.07,0.05$ and $-0.05 \mathrm{~m} \mathrm{~s}^{-2}$. iEMG of the TA in the burst at heel contact was normalized to the value at step 0 in the WRT (with corresponding acceleration) for each subject. iEMG-values in the graphs represent the mean and standard deviation of all normalized averages of the subjects. Statistics were only applied in the zone step -1 to step +1 , as that is the region of interest. To give the reader a clear view on the data, however, the progress of iEMG is given from step -5 to step +5 with $R^{2}$ values indicating the accuracy of the regression line. 


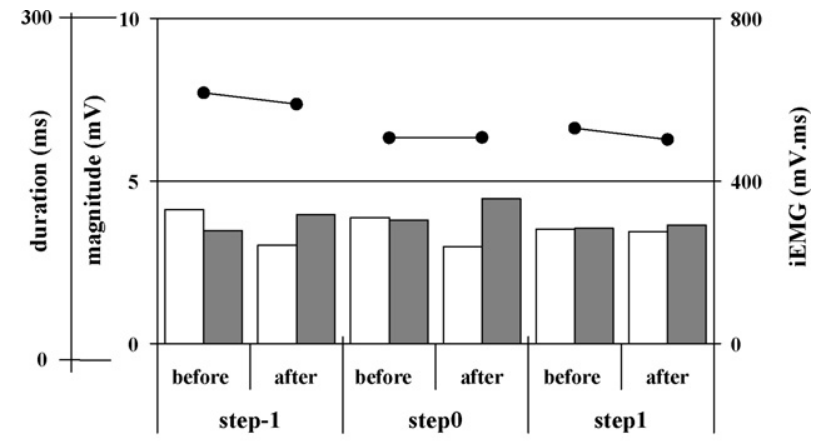

(a)
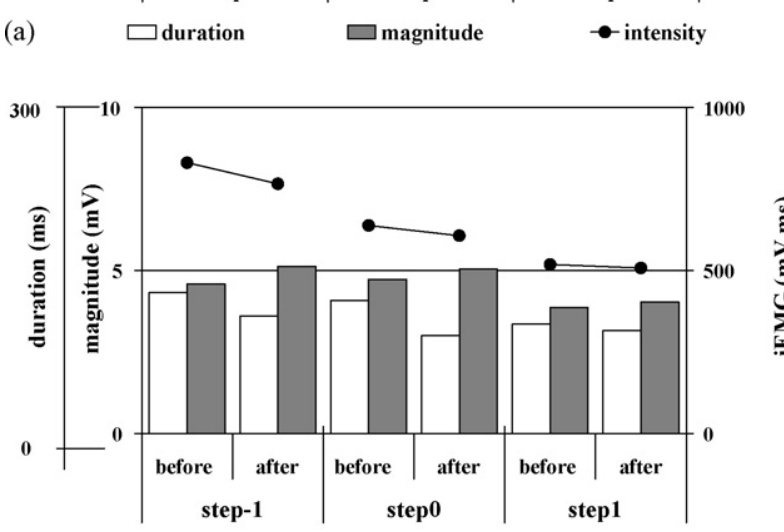

(b)

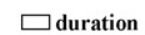

$\square$ magnitude

$\bullet$ - intensity

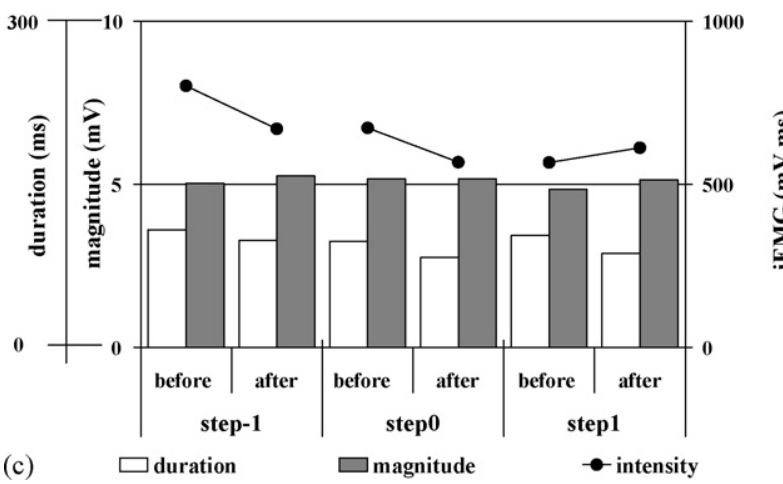

Fig. 2. Integrated EMG (iEMG) of the tibialis anterior before and after fatigue in the WRT, $a=0.10,0.07$ and $0.05 \mathrm{~m} \mathrm{~s}^{-2}$.

did not affect iEMG. The $\eta^{2}$-value, which explains the importance of the fatigue effect, was small (0.02). No further analyses were undertaken to reveal effects of fatigue in step +1 .

The duration of the burst was significantly lower after fatigue in step -1 and step 0 , with a difference between accelerations (Table 4) but no interaction between fatigue and acceleration. In step +1 , the duration of the burst was affected neither by fatigue of the TA nor by the rate of acceleration. Furthermore, there was no interaction-effect between fatigue and acceleration (Table 4).

After fatigue, the magnitude of the burst increased in step -1 and step 0 , whereas no effects were found in step +1 (Table 4). The acceleration, on the other hand, affected the magnitude of the signal in all three steps (Table 4). No interaction between acceleration and EMG-signal was found in step -1 and step 0 .

\subsection{2. $R W T$}

In step -1 , there was one main effect of acceleration on $\operatorname{iEMG}\left(F_{2,24}=6.43, p=0.01, \eta^{2}=0.35\right)$ and a main effect of both acceleration $\left(F_{2,24}=15.48, p<0.01, \eta^{2}=0.56\right)$ and fatigue $\left(F_{1,12}=5.26, p=0.04, \eta^{2}=0.31\right)$ on magnitude of the burst. In step +1 there was a main effect of acceleration on magnitude $\left(F_{2,24}=9.33, p=0.01, \eta^{2}=0.44\right)$ and duration of the burst $\left(F_{2,24}=5.69, p=0.03, \eta^{2}=0.32\right)$.

No interaction-effects between fatigue and acceleration were found. $\eta^{2}$ Values were lower for the RWT compared to the WRT.

\subsection{Spatiotemporal factors}

\subsubsection{WRT}

SF was significantly lower after fatigue in the transition step $\left(F_{1,12}=7.09, p=0.026, \eta^{2}=0.441\right)$. In step -1 and step +1 , SF remained unchanged (Fig. 3a).

There was a main effect of fatigue on SL in step -1 $\left(F_{1,12}=6.12, \quad p=0.033, \quad \eta^{2}=0.380\right)$ and in step +1 $\left(F_{1,12}=7.74, p=0.019, \eta^{2}=0.436\right)$ with SL significantly lower after fatigue. There were no main effects or interaction-effects on SL in the transition step (Fig. 3a).
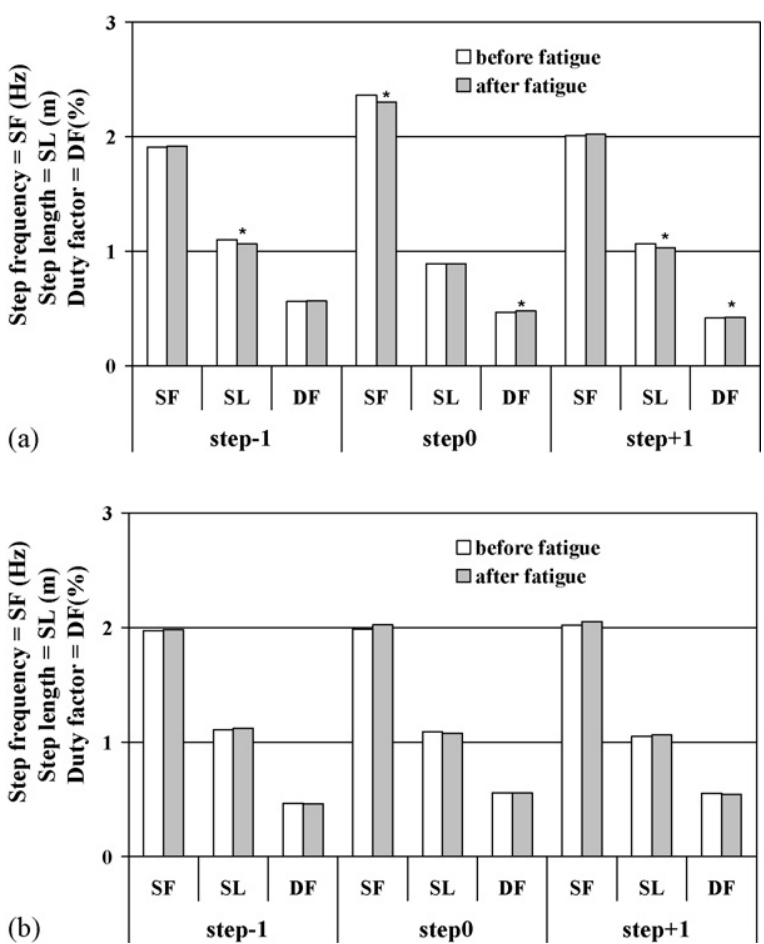

Fig. 3. Spatiotemporal factors before and after fatigue in: (a) WRT and (b) RWT. Asteriskes indicate significant differences $\left({ }^{*} p<0.05\right)$. There are no significant differences in spatiotemporal factors between the accelerations (no differences in transition speed either). Therefore, no distinction is made between the accelerations. Bars represent the average of (a) all accelerations in the WRT-protocol and (b) all decelerations in the RWT-protocol. 
DF in step -1 was not changed, neither by the degree of acceleration nor by muscular fatigue. In step 0 , a main effect of acceleration $\left(F_{2,24}=4.55, p=0.036, \eta^{2}=0.452\right)$ and fatigue $\left(F_{1,12}=8.85, p=0.012, \eta^{2}=0.424\right)$ on DF was found and no interaction between both. In step $+1 \mathrm{DF}$ was lower after fatigue $\left(F_{1,12}=7.24, p=0.020, \eta^{2}=0.376\right)$.

\subsection{2. $R W T$}

In step -1 no effects were found for SF (Fig. 3b). In step 0 an interaction-effect of acceleration and fatigue was found $\left(F_{2,24}=15.47, \quad p=0.001, \quad \eta^{2}=0.738\right)$. The degree of acceleration had a main effect on $\mathrm{SF}$ in step 1 $\left(F_{2,24}=7.22, p=0.010, \eta^{2}=0.568\right)$.

SL was unchanged in step -1 and step 0: no main or interaction-effects were found for acceleration and fatigue. In step 1 an interaction-effect was found $\left(F_{2,24}=4.75\right.$, $p=0.033, \eta^{2}=0.463$ ).

For DF a main effect of acceleration was found in step -1 $\left(F_{2,24}=8.19, \quad p=0.007, \quad \eta^{2}=0.598\right) \quad$ and $\quad$ step +1 $\left(F_{2,24}=6.94, p=0.011, \eta^{2}=0.558\right)$.

\subsection{Foot angle}

To explore possible influences of muscular fatigue of the TA on the foot angle (shoe sole-ground), foot angle was calculated for five randomly selected subjects. As can be seen in Table 5, foot angle decreased after WRT. No differences for fatigue were observed.

\section{Discussion}

Our main hypotheses were confirmed. (1) The WRTspeed was significantly decreased after fatigue of TA. RWTspeed did not change except for the intermediate deceleration. (2) In the WRT, iEMG increased with increasing walking speed, then decreased after the transition to the running gait. The third hypothesis was only partially confirmed as (3) in the RWT, no effect on iEMG was found for fatigue. However, an effect of transition was observed as iEMG increased during the transition step, and then decreased with decreasing walking speed.

\subsection{WRT}

WRT-speed was lower after fatigue of the TA (Table 3). The decrease of WRT-speed with fatigue was related to a significant decrease in SL (Fig. 3a). SF, on the other hand, remained constant after fatigue, except for the transition step. DF was higher in the transition step and the first running step, indicating that there was a longer contact with the ground. Segers et al. [21] described the evolution of the spatiotemporal parameters in a protocol with gradually changing speed and identified the WRT-step as an outlier. As can be seen in Fig. 3, SF of the transition step still differed from the previous walking steps and the following running
Table 3

Transition speed before and after fatigue for all accelerations (mean \pm stanstandard deviation)

\begin{tabular}{cll}
\hline Acceleration $\left(\mathrm{m} \mathrm{s}^{-2}\right)$ & \multicolumn{2}{l}{ Transition speed $\left(\mathrm{m} \mathrm{s}^{-1}\right)$} \\
\cline { 2 - 3 } & Before fatigue & After fatigue \\
\hline WRT & $2.16 \pm 0.12$ & $2.06 \pm 0.07^{* *}$ \\
0.1 & $2.10 \pm 0.06$ & $2.00 \pm 0.07^{* *}$ \\
0.07 & $2.12 \pm 0.08$ & $2.04 \pm 0.09^{* *}$ \\
0.05 & & \\
RWT & $2.19 \pm 0.14$ & $2.19 \pm 0.14$ \\
-0.1 & $2.12 \pm 0.09$ & $2.20 \pm 0.14^{* *}$ \\
-0.07 & $2.17 \pm 0.06$ & $2.18 \pm 0.06$ \\
-0.05 & & \\
\hline
\end{tabular}

Significant difference between transition speed before and after fatigue ** $p<0.01$.

steps after fatigue and was the only step where speed was altered by a decrease in SF.

The pattern of the iEMG followed the hypothesized pattern for the TA. iEMG increased before transition, then dropped to a lower value after transition (Fig. 1). The amplitude and duration of the isolated burst of concentriceccentric activity of the TA around the heel contact reduced after transition (Fig. 2), reinforcing the theory of Hreljac et al. [2]. TA fatigue would cause a feeling of local discomfort probably leading to transition [2].

Hreljac linked his 'maximal stress theory' to the maximum amplitude of the EMG-signal. Therefore, the maximum amplitude of the rectified EMG-signal (before fatigue) was studied for five subjects. This confirmed the findings of Hreljac et al. [2]. The maximum amplitude was lower after the transition to running gait. Despite this finding, iEMG provided more information about the RPE. Since RPE consists of a central and a local factor [14], localized muscular overexertion of the TA was greater during walking due to the periodic burst of high activity in comparison to the longer periodic burst of moderate activity in running [2]. Peak EMG, however, does not fully provide the information needed to describe a periodic burst of TA activity as it is momentary information. iEMG provides more complete information about burst activity, and indicated that the burst around heel contact was indeed lower after transition to running.

One of the most remarkable differences between walking and running at speeds in proximity of the transition-speed was the touch-down angle of the foot that was smaller during running (Table 5, literature $[18,26]$ ). It is expected that this would introduce a less demanding situation for TA.

After fatigue, there was a decrease in iEMG related to the duration of the burst (Fig. 2; Table 4). The mean amplitude of the burst was higher but would not compensate for the decrease in duration. After fatigue, a similar evolution of iEMG in the approach to the WRT and a decrease in maximum amplitude after the transition was found. Muscular fatigue is known to increase the sense of effort 
Table 4

Main and interaction-effects found by the repeated measures analysis for step -1 , step 0 and step +1 in the WRT

\begin{tabular}{|c|c|c|c|c|c|c|c|c|c|}
\hline & \multicolumn{3}{|c|}{ Acceleration } & \multicolumn{3}{|l|}{ Fatigue } & \multicolumn{3}{|c|}{ Interaction } \\
\hline & $F(2,24)$ & $p$ & $\eta^{2}$ & $F(1,12)$ & $p$ & $\eta^{2}$ & $F(2,24)$ & $p$ & $\eta^{2}$ \\
\hline \multicolumn{10}{|l|}{$i E M G$} \\
\hline Step -1 & 7.69 & 0.00 & 0.40 & 4.60 & 0.05 & 0.28 & 0.02 & 0.98 & 0.00 \\
\hline Step 0 & 1.09 & 0.35 & 0.08 & 22.84 & 0.00 & 0.66 & 0.12 & 0.89 & 0.01 \\
\hline Step +1 & 1.77 & 0.21 & 0.13 & 0.28 & 0.76 & 0.02 & 4.03 & 0.03 & 0.25 \\
\hline \multicolumn{10}{|l|}{ Duration } \\
\hline Step -1 & 4.75 & 0.02 & 0.28 & 13.64 & 0.00 & 0.53 & 2.86 & 0.08 & 0.19 \\
\hline Step 0 & 4.48 & 0.02 & 0.27 & 13.84 & 0.00 & 0.54 & 0.41 & 0.67 & 0.03 \\
\hline Step +1 & 2.83 & 0.12 & 0.19 & 1.62 & 0.22 & 0.12 & 0.21 & 0.81 & 0.02 \\
\hline \multicolumn{10}{|l|}{ Magnitude } \\
\hline Step -1 & 31.97 & 0.00 & 0.73 & 21.11 & 0.00 & 0.64 & 3.04 & 0.07 & 0.20 \\
\hline Step 0 & 5.62 & 0.01 & 0.32 & 19.11 & 0.00 & 0.61 & 2.47 & 0.11 & 0.17 \\
\hline Step +1 & 5.66 & 0.03 & 0.33 & 0.04 & 0.97 & 0.00 & 5.61 & 0.01 & 0.32 \\
\hline
\end{tabular}

in the muscle [19], probably causing the decrease in WRTspeed. After fatigue, the force-generating capacity, needed to position the foot in a controlled manner and prevent "slapping" [27-30], was reduced.

The fact that the touch-down angle of the foot was lower during running (Table 5), may indicate a tendency to change the mode of locomotion sooner following TA fatigue in order to avoid the larger eccentric action needed to lower the foot during walking.

TA fatigue influences the loading rate of the vertical GRF in steady-state running $[30,31]$. An increased loading rate is also one of the typical characteristics of the last steps before the (WRT) in a protocol with gradually increasing speed [4]. This may mean that loading rate of GRF is the link between TA fatigue and lower WRT-speed.

Another possible explanation could be the greater instability of the foot after TA fatigue [29,30]. At heel contact, the eversion load is maximized in walking [27,30]. If foot eversion is insufficiently counteracted by the TA, with secondary function preventing eversion $[27,30]$, this would cause a medial shift of the centre of pressure and a lateral shift of the centre of mass [29,30]. It may be that an earlier transition to running is linked to this subjective feeling of instability.

We wish to emphasize, however, that the TA signal is only one among numerous gait determinants and could be overruled by other factors, such as fear or visual flow $[33,34]$.

Table 5

Touch-down angle of the foot (shoe sole/ground) in the WRT

\begin{tabular}{lll}
\hline & \multicolumn{2}{l}{$\begin{array}{l}\text { Foot angle }\left(a=0.10 \mathrm{~m} \mathrm{~s}^{-2},\right. \\
\end{array}$} \\
\cline { 2 - 3 } & Before fatigue & After fatigue \\
\hline Walking steps & $34.92 \pm 2.57$ & $32.32 \pm 1.99$ \\
Transition step & $23.56 \pm 2.57^{*}$ & $25.85 \pm 2.38^{*}$ \\
Running steps & $24.15 \pm 2.15^{*}$ & $22.74 \pm 1.78^{*}$ \\
\hline
\end{tabular}

\footnotetext{
* Significant difference $(p<0.05)$ with foot angle during walking steps.
}

\section{2. $R W T$}

RWT-speed was altered after fatigue in the intermediate deceleration. The interaction-effects between fatigue and acceleration can be explained by this difference between decelerations. This discrepancy could be due to the fact that the RWT is less urgent than the WRT [7]. In the WRT there is local discomfort in the dorsiflexor area whereas in the RWT there is no such feeling of discomfort [3] If desired, subjects could run at a lower speed, whereas the occurrence of the WRT is limited. Therefore, variability in RWT through conscious decision making may be higher (Table 2) [3].

The protocol of gradually changing speed allowed us to describe RWT, in contrast to some previous studies $[1-3,5,9,10]$. As can be seen in Fig. 1, iEMG increased after transition, mostly due to an increase in the duration of the burst before heel contact. Fatigue of the TA did not change iEMG. The pattern of the iEMG indicated that TA is probably not a determining factor for the RWT, as in walking it reaches a higher activation level, which may be associated with the larger touch-down angle of the foot (Table 5).

In future research, the transition phenomenon (WRT and RWT) in an overground condition should be studied. In the present study walking/running on a treadmill was chosen because of the protocol with gradually changing speed. It could be that transition-speed is changed by the use of the treadmill $[33,39]$ because: (1) the treadmill induces changes in kinematic variables which are associated to gait transition, i.e. foot and ankle angle [35,36], (2) power flows from the athlete to the treadmill and vice versa [35,37] and (3) spatiotemporal characteristics are influenced by the treadmill [36,38]. Furthermore, studying transition overground enables ground reaction force measurements using the standard technology of force plates. Inverse dynamics then allows for calculation of the net joint powers which are associated to muscular activity levels. 
Hreljac [5] showed that, at transition, critical levels of ankle angular velocities and accelerations are reached. According to Hreljac et al. [2], the dorsiflexors would work at their maximal capacity to produce the required large angular accelerations and changing gait (WRT) would alleviate the locally perceived exertion on the dorsiflexors. Recent modelling by Neptune and Sasaki [40], however, showed that the plantar flexors are important determinants of the WRT. Taken these two opinions into account and knowing that the ankle moment is the sum of the moments about the ankle joint generated by forces developed by both agonists and antagonists, it could be stipulated that the present study results are influenced by altered antagonist activity.

The main finding of our study was that WRT-speed is lower after fatigue, whereas RWT-speed is not altered. Integrated EMG in the WRT is lower after transition to the running gait, reinforcing the theory of Hreljac et al. [2] in a protocol with gradually changing speed. TA is a probable factor determining the WRT. In the RWT no differences were found, which indicates that WRT and RWT are two different mechanisms.

\section{Acknowledgements}

This research was supported by BOF-RUG B/03796/01IV1. The authors acknowledge Ing. P. Van Cleven and Ing. D. Spiessens for data collection and technical support and Lic. A. De Cock and Dr. J. Vanrenterghem for their professional advice.

The authors also thank Prof. Dr. D. Adriaens (Ghent University, department of Vertebrate Morphology \& Zoology Museum) for the use of the JVC highspeed camera.

\section{References}

[1] Hreljac A. Determinants of the gait transition speed during human locomotion: kinetic factors. Gait Posture 1993;1:217-23.

[2] Hreljac A, Arata A, Ferber R, Mercer JA, Row BR. An electromyographical analysis of the role of the dorsiflexors on the gait transition during human locomotion. J Appl Biomech 2001;17: 287-96.

[3] Prilutsky BI, Gregor RJ. Swing- and support related muscle actions differentially trigger human walk-run and run-walk transitions. J Exp Biol 2001;204:2277-87.

[4] Li L, Hamill J. Characteristics of the vertical ground reaction force component prior to gait transition. Res Q Exercise Sport 2002;73:22937.

[5] Hreljac A. Determinants of the gait transition speed during human locomotion: kinematic factors. J Biomech 1995;28:669-77.

[6] Raynor AJ, Yi CJ, Abernethy B, Jong QJ. Are transitions in human gait determined by mechanical, kinetic or energetic factors? Hum Mov Sci 2002:21:785-805.

[7] Hanna A, Abernethy B, Neal RJ, Burgess-Limerick R. Triggers for the transition between human walking and running. In: Sparrow WA, editor. Energetics of human activity. Champaign, IL: Human Kinetics; 2000. p. 124-64.
[8] Mercier J, Le Gallais D, Durrand M, Goudal C, Micallef JP, Préfaut C. Energy expenditure and cardiorespiratory responses at the transition between walking and running. Eur J Appl Physiol 1994;65:525-9.

[9] Hreljac A. Preferred and energetically optimal gait transition speeds in human locomotion. Med Sci Sports Exer 1993;25:1158-62.

[10] Hreljac A, Parker D, Quintana R, Abdala E, Sison M. Energetics and perceived exertion of low speed running and high speed walking. Facta Universitatis Phys Educ Sport 2002;1:27-35.

[11] Brisswalter J, Mottet D. Energy cost and stride duration variability at preferred transition gait speed between walking and running. Can J Appl Physiol 1996;21:471-80.

[12] Minetti AE, Ardigo LP, Saibene F. The transition between walking and running in humans: metabolic and mechanical aspects at different gradients. Acta Physiol Scand 1994;150:315-23.

[13] Tseh W, Bennett J, Caputo JL, Morgan DW. Comparison between preferred and energetically optimal transition speeds in adolescents. Eur J Appl Physiol 2002;88:117-21.

[14] Noble BJ, Metz KF, Pandolf KB, Cafarelli E. Perceptual responses to exercise: a multiple regression study. Med Sci Sport Exer 1973;6: 104-9.

[15] Thorstensson A, Roberthson H. Adaptations to changing speed in locomotion: speed of transition between walking and running. Acta Physiol Scand 1987;131:211-4.

[16] McCloskey DI, Gandevia S, Potter EK, Colebatch JG. Muscle sense and effort: motor commands and judgments about muscular contractions. Adv Neurol 1983;39:151-67.

[17] Hampson DB, St Clair Gibson A, Lambert MI, Noakes TD. The influence of sensory cues on the perception of exertion during exercise and central regulation of exercise performance. Sports Med 2001;31: 935-52.

[18] Nilsson J, Thorestensson A, Halbertsma J. Changes in leg movement and muscle activity with speed of locomotion and mode of progression in humans. Acta Physiol Scand 1985;123:457-75.

[19] Kant-Braun JA, Ng AV, Doyle JW, Towse TF. Human skeletal muscle responses vary with age and gender during fatigue due to incremental isometric exercise. J Appl Physiol 2002;93:1813-23.

[20] Hreljac A. Effects of physical characteristics on the gait transition speed during human locomotion. Hum Mov Sci 1995;14:205-16.

[21] Segers V, Aerts P, Lenoir M, De Clercq D. Spatiotemporal characteristics of the walk-to-run and run-to-walk transition when gradually changing speed. Gait Posture 2006;24:247-54.

[22] Li L. Stability landscapes of walking and running near gait transition speed. J Appl Biomech 2000;16:428-35.

[23] Wall JC, Charteris J. The process of habituation to treadmill walking at different velocities. Ergonomics 1980;23:425-35.

[24] Borg G. Borg's perceived exertion and pain scales Champaign, IL: Human kinetics; 1998.

[25] Zatsiorsky VM, Werner SL, Kaimin MA. Basic kinematics of walking. Step length and step frequency. a review. J Sport Med and Phys Fit 1994;34:109-34.

[26] Sasaki K, Neptune RR. Differences in muscle function during walking and running at the same speed. J Biomech 2006;39:2005-13.

[27] Perry J. Gait analysis: normal and pathological function. SLACK incorporated 1992;51-89 [Chapter 4].

[28] Flynn JM, Holmes JD, Andrews DM. The effect of localized muscle fatigue on tibial impact acceleration. Clin Biomech 2004;19: 726-32.

[29] Gefen A. Simulations of foot stability during gait characteristic of ankle dorsiflexor weakness in the elderly. IEEE T Neur Sys Reh 2001;9:333-7.

[30] Cole GK, Nigg BM, van den Bogert AJ, Gerritsen KGM. Lower extremity joint loading during impact in running. Clin Biomech 1996;4:181-93.

[31] Christina KA, White SC, Gilchrist LA. Effect of localized fatigue on vertical ground reaction forces and ankle joint motion during running. Hum Mov Sci 2001;20:257-76. 
[33] Malcolm P, Lenoir M, Aerts P, Segers V, De Clercq D. Treadmill versus overground run to walk and walk to run transition speed in unsteady state locomotion conditions. In: XXth congress of the international society of biomechanics; 2005.

[34] Mohler BJ, Thompson WB, Creem-Regehr S, Pick HL. Visual motion influences locomotion in a treadmill virtual environment. ACM SIGGRAPH 2004;19-22.

[35] Savelbergh HHCM, Vorstenbosch MATM, Kamman EH, van de Weijer JGW. Schambardt. Intra-stride belt-speed variation affects treadmill locomotion. Gait Posture 1998;7:26-34.

[36] Wank V, Frick U, Schmidtbleicher D. Kinematics and electromyography of lower limb muscle in overground and treadmill running. Int J Sports Med 1998;19:455-61.
[37] Shamhart HC, van den Bogert AJ, Lammertink JLMA. Power transfer from treadmill to athlete. In: Proceedings of the eighth biennial conference of the Canadian society for biomechanics; 1994. p. 306-7.

[38] Stolze H, Kuhtz-Buschbeck, Mondwurf C, Boczek-Funcke A, Jönck K, Deuschl G, Illert M. Gait analysis during treadmill and overground locomotion in children and adults. Electroenceph Clin Neurophysiol 1997;10:490-7.

[39] Johnson JL, Li L. Gait transition between walking and running, comparing treadmill to ground locomotion. J Sports Exer Psych 2000;22S:56.

[40] Neptune RR, Sasaki K. Ankle plantar flexor production is an important determinant of the preferred walk-to-run transition speed. J Exp Biol 2005;208:799-808. 